
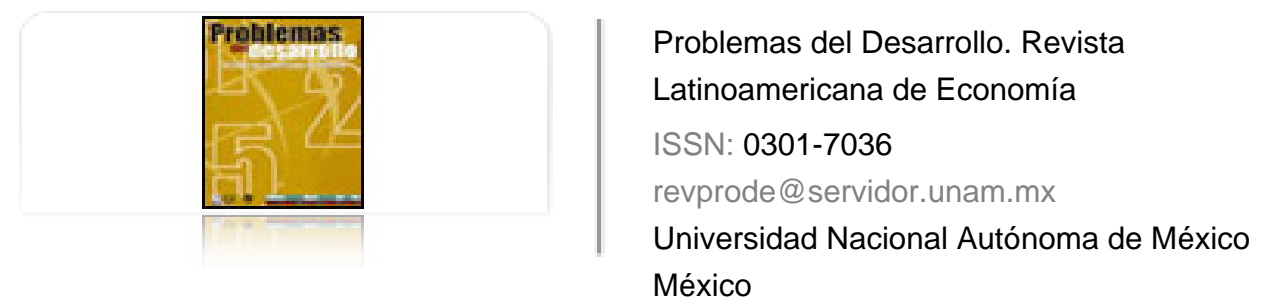

Bastian, Eduardo; Soihet, Elena

Argentina y Brasil: desafíos macroeconómicos

Problemas del Desarrollo. Revista Latinoamericana de Economía, vol. 43, núm. 171, octubre-

diciembre, 2012, pp. 83-109

Universidad Nacional Autónoma de México

Distrito Federal, México

Disponible en: http://www.redalyc.org/articulo.oa?id=11823951005

- Cómo citar el artículo

- Número completo

- Más información del artículo

Página de la revista en redalyc.org

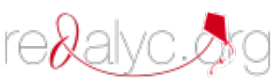

Sistema de Información Científica

Red de Revistas Científicas de América Latina, el Caribe, España y Portugal

Proyecto académico sin fines de lucro, desarrollado bajo la iniciativa de acceso abierto 


\title{
Argentina y Brasil: Desafíos MACroECONÓMICOS ${ }^{1}$
}

\author{
Eduardo Bastian* y Elena Soihet**
}

Fecha de recepción: 25 de septiembre de 2011. Fecha de aceptación: 19 de marzo de 2012.

\begin{abstract}
RESUMEN
En la década de 1990, Argentina y Brasil siguieron estrategias económicas parecidas. Sin embargo, las crisis cambiarias que Brasil sufrió en 1998-1999 y la Argentina en 20012002, acabaron siendo un punto de inflexión, ya que después de las crisis los dos países optaron por regímenes macroeconómicos muy diferentes. Este artículo tiene como objetivo discutir las experiencias de Argentina y Brasil bajo estos distintos regímenes macroeconómicos. Particularmente queremos analizar: 1) las principales características de los dos regímenes macroeconómicos; 2) las razones que llevaron a los argentinos y los brasileńos a optar por estrategias diferentes después de sus respectivas crisis cambiarias; 3) los resultados alcanzados por los dos países en la década pasada; 4) los principales desafíos que estos dos regímenes enfrentan hoy.
\end{abstract}

Palabras clave: Argentina, Brasil, regímenes macroeconómicos, inflación, crecimiento.

\section{Argentina and Brazil: Macroeconomic Challenges}

\begin{abstract}
In the 1990s, Argentina and Brazil followed similar economic strategies. However, the currency crisis that Brazil went through in 1998-1999 and Argentina suffered in 20012002 served as an inflection point, because after these crises, both countries opted for very different macroeconomic regimes. This article seeks to discuss the experiences of Brazil and Argentina under different macroeconomic regimes. Specifically, we aim to analyze the following: 1) the key characteristics of both macroeconomic regimes; 2) the reasons why Argentina and Brazil opted for different strategies following their respective currency crises; 3) the results obtained by both countries in the past decade; 4) the main challenges that these two regimes face today.
\end{abstract}

Key Words: Argentina, Brazil, macroeconomic regimes, inflation, growth.

1 Los autores agradecen los comentarios y sugerencias de André Modenesi, Antonio Licha, Federico Pastrana, Franklin Serrano, Luiz Fernando de Paula y Ricardo Summa, eximiéndolos, por supuesto, de cualquier responsabilidad por el contenido de la obra final. Los autores también agradecen a Margarida Gutiérrez por proporcionar datos sobre los cambios recientes en el mercado brasileño de divisas. Por último, agradecemos también a los árbitros anónimos de la revista por sus comentarios y sugerencias.

* Profesor adjunto del Instituto de Economía de la Universidade Federal de Rio de Janeiro (IE/UfrJ). Correo electrónico: eduardobastian@ie.ufrj.br

** Profesora adjunta de la Universidade Federal Rural de Rio de Janeiro (IM/UfrRJ). Correo electrónico: e.soihet@globo.com 


\section{ARGENTINE ET BRÉSIL: DÉFIS MACROÉCONOMIQUES \\ Résumé}

Durant la décennie des années 1990, l'Argentine et le Brésil ont appliqué des stratégies économiques semblables. Néanmoins, les crises du système de change subies par le Brésil en 1998-1999 et par l'Argentine en 2002-2002 ont finalement constitué un point d'inflexion, étant donné qu'après ces crises les deux pays ont opté pour des régimes macroéconomiques distincts. Cet article a pour but de discuter les expériences de l'Argentine et du Brésil sous ces différents régimes macroéconomiques. Nous souhaitons notamment analyser 1) les principales caractéristiques des deux régimes macroéconomiques, 2) les raisons qui ont mené les Argentins et les Brésiliens à opter pour des stratégies différentes après leurs respectives crises du système de change, 3 ) les résultats obtenus par les deux pays durant la dernière décennie, et 4) les principaux défis que ces deux régimes affrontent aujourd'hui.

Mots clés : Argentine, Brésil, régimes macroéconomiques, inflation, croissance.

\section{ARGENTINA E BRASIL: DESAFIOS MACROECONÓMICOS \\ Resumo}

Na década de 1990, Argentina e Brasil seguiram estratégias económicas parecidas. Porém, as crises cambiarias que Brasil sofreu em 1998-1999 e a Argentina em 2001-2002, acabaram sendo um ponto de inflexão, já que depois das crises os dois países optaram por regimes macroeconómicos muito diferentes. Este artigo tem como objetivo discutir as experiências de Argentina e Brasil sob estes distintos regimes macroeconômicos. Particularmente queremos analisar: 1) as principais características dos dois regimes macroeconómicos; 2) as razoes que levaram aos argentinos e aos brasileiros a optar por estratégias diferentes depois de suas respectivas crises cambiarias; 3) os resultados alcançados pelos dois países na década passada; e 4) os principais desafios que estes dois regimes enfrentam hoje.

Palavras-chave: Argentina, Brasil, regimes macroeconômicos, inflação, crescimento.

阿根廷和巴西：宏观经济制度的目标、效果和挑战

\section{摘要}

上世纪90年代，阿根廷和巴西采取了类似的经济战略。然而，巴西和阿根 廷分别于 1998-1999年和2001-2002年经历的货币危机成为了两国政策 变化的转折点, 因为在危机之后，两国选择了非常不同的宏观经济制度。 本文试图讨论巴西和阿根廷在不同宏观经济制度下的经验。具体而言，我 们的目标是分析以下问题：1)两国宏观经济制度的关键特征；2)阿根廷 和巴西在危机之后采取不同战略的原因；3）两国在过去十年中取得的成 效; 4)目前两国的宏观经济制度面临的挑战。 关键词：阿根廷巴西 宏观经济制度 通货膨胀增长 


\section{INTRODUCCIÓN}

En la década de 1990, Argentina y Brasil siguieron estrategias económicas parecidas. Por un lado, después de años de inflación alta, ambos países alcanzaron la estabilidad de precios con planes basados en alguna forma de ancla cambiaria. ${ }^{2}$ Por otro lado, además de las políticas de estabilización parecidas, los dos países acabaron adoptando en esta misma década, una serie de reformas liberalizadoras en la línea de las directrices sugeridas por el Consenso de Washington. Estas reformas fueron un fracaso en términos de crecimiento económico y, lo que es peor, la adopción de anclas cambiarias acabó por dejar a los países extremadamente vulnerables a ataques especulativos. El resultado fue que los brasileños y los argentinos sufrieron crisis cambiarias al final de la década de los años noventa y principio del siglo XXI.

Las crisis cambiarias marcaron una transformación en la tendencia observada en los años de 1990. En otras palabras, Brasil y Argentina adoptaron estrategias diferentes después de sus respectivas crisis. Argentina enfatizó el crecimiento económico y el desarrollo industrial partiendo de un régimen macroeconómico centralizado en un tipo de cambio estable y competitivo. En Brasil se dio prioridad a la estabilidad de precios con base en un trípode de metas de inflación, cambio flexible y metas de superávit primario en las cuentas fiscales.

Así, el artículo presente tiene el objetivo de discutir, exactamente, las diferencias entre los regímenes adoptados por Argentina y Brasil después de sus respectivas crisis cambiarias. En particular, queremos analizar: 1) las principales características de los dos regímenes macroeconómicos; 2) las razones que llevaron a los argentinos y los brasileńos a optar por estrategias diferentes; 3) los resultados alcanzados en la década pasada; 4) los principales desafíos que estos dos regímenes enfrentan hoy.

El artículo está dividido en tres secciones, además de la introducción y la conclusión. La primera sección se destina a presentar los dos regímenes macroeconómicos. La segunda sección discute las razones por las cuales Argentina y Brasil optaron por regímenes macroeconómicos diferentes, y también presenta los resultados alcanzados analizando los méritos y las fragilidades de ambos regímenes macroeconómicos. Por fin, la tercera sección contiene una evaluación general de los resultados, y discute los desafíos económicos actuales de Argentina y Brasil y el futuro de los respectivos regímenes macroeconómicos.

2 A pesar de este punto de similitud, Argentina optó por una forma más radical que Brasil, pues adoptó el régimen de currency-board, mientras que Brasil siguió un régimen de bandas cambiarias. 


\section{REGÍMENES MACROECONÓMICOS DE BRASIL Y ARGENTINA DESPUÉS DE LAS CRISIS CAMBIARIAS: UNA VISIÓN SINTETIZADA}

\section{El régimen macroeconómico brasileño (1999-2010)}

Después de abandonar el ancla cambiaria en enero de 1999, tras un ataque especulativo, el gobierno brasileño adoptó, en junio de aquel año, el régimen de metas de inflación. El cambio de régimen no significó, sin embargo, una ruptura con la ortodoxia económica. El régimen de metas de inflación es una estructura institucional que está basada en los presupuestos de la ortodoxia los cuales, entre otras cosas, establecen que: 1 ) no hay, a largo plazo, un trade-off entre producto (empleo) e inflación; 2) un ancla nominal es un elemento clave para garantizar buenos resultados por parte de la política monetaria ${ }^{3}$ (Mishkin, 2007: 1). De esta forma, el mérito del régimen de metas de inflación sería funcionar como un ancla nominal mejor que otros regímenes monetarios (Mishkin, 2007: 11). Así, en el fondo, la sustitución del ancla cambiaria por el régimen de metas de inflación fue simplemente un cambio en el ancla nominal de la economía brasileña.

Según Bogdanski et al. (1999) y Barbosa-Filho (2009), el régimen de metas para la inflación adoptado en Brasil tiene, entre otras cosas, la siguiente configuración: 1) para determinar el cumplimiento de las metas se eligió el Índice de Precios al Consumidor Amplio (IPCA), calculado por el IBGE, sin excluir los precios más inestables; 2) las metas incluyeron un intervalo variable de tolerancia para arriba y para abajo (inicialmente de $2 \%$, aumentado para $2.5 \%$ y nuevamente reducido para $2 \%$ en 2006); 3) las metas y los intervalos de tolerancia serían fijados por el Consejo Monetario Nacional (CMN), ${ }^{5}$ con una antecedencia de un año y medio; 4) la meta fijada debería ser cumplida en

3 Un ancla nominal es "una variable nominal que la autoridad monetaria usa para atar los niveles de precio como, por ejemplo, la tasa de inflación, el tipo de cambio o la oferta de moneda" (Mishkin, 2007: 4).

4 Según Mishkin (2007), tendría las siguientes ventajas entre otras cosas: 1) fácil de ser entendido por el público; 2) aumento de accountability del banco central, ya que debe alcanzar una meta determinada previamente especificada y conocida por los agentes; 3) coloca en el centro del debate sobre política monetaria aquello que el banco central consigue hacer mejor a largo plazo, o sea, controlar la inflación; 4) no existe la necesidad de una relación estable entre moneda e inflación, a diferencia de lo que sucede en el caso de las metas monetarias (Mishkin, 2007: 12-13).

5 Formado por los ministros de Hacienda y de Planeamiento y por el presidente del Banco Central. 
el transcurso de doce meses, año calendario; 5) en el caso de no ser cumplida la meta, el presidente del Banco Central del Brasil tiene que escribir una carta abierta al ministro de Economía explicando las causas de no haber alcanzado la meta (Bogdanski et al., 2000: 11; Barbosa-Filho, 2009: 140-141).

Por otro lado, desde el punto de vista del régimen fiscal, la idea era obtener -a través de la disciplina fiscal- mayores superávits primarios/PIB, para permitir así la reducción de la Deuda Líquida del Sector Público/PIB, el aumento de los plazos de la deuda y el aumento de participación de los papeles prefijados. Este nuevo régimen fiscal inaugurado en 1999 sería complementado con la Ley de Responsabilidad Fiscal (Ley Complementaria del 4 de mayo de 2000), la cual restringió la capacidad de los gobiernos estatales y municipales de solicitar préstamos, estableciendo límites para gastos con empleados y para la deuda pública. Estas medidas mostrarían el compromiso con la solvencia fiscal.

Finalmente, las políticas macroeconómicas brasileñas se complementaron con el tipo de cambio flexible. Después de las crisis cambiarias al final de los años 1990, creció la idea de que los regímenes de cambio fijo dejan a los países mucho más vulnerables a fugas de capitales eventuales, obligándolos a acumular reservas cambiarias. De esta forma, para eliminar los riesgos asociados al cambio fijo y para independizar la política monetaria, se optó por un régimen de flexibilidad cambiaria.

Así, en síntesis, el régimen macroeconómico brasileño post-1999 está formado por un trípode constituido por metas de inflación, cambio flexible y superávits primarios en las cuentas públicas.

\section{El régimen macroeconómico argentino (2003-2010)}

El régimen macroeconómico seguido por la Argentina a partir de 2002 fue muy diferente de la estrategia seguida por los brasileños. Después de la crisis de la convertibilidad, el principal objetivo pasó a ser maximizar el crecimiento económico, la creación de empleos y la recuperación de la industria, sin que la estabilidad de precios fuese considerada sine qua non para esta finalidad. Así, en líneas generales, puede afirmarse que el régimen macro argentino se sostiene en algunos pilares como:

"un tipo de cambio competitivo (estable), superávit fiscal y en la cuenta corriente de la balanza de pagos, y una política monetaria que buscaba al mismo tiempo evitar la apreciación del tipo de cambio real, acumular reservas internacionales, facilitar la recuperación de la liquidez y... limitar por medio de acciones de esterilización el efecto monetario expansivo creado -a partir de la mitad del 2002- por la acumulación de reservas" (Damill \& Frenkel, 2009: 1-2). 
Concretamente, la política fiscal estaba dirigida a obtener superávit fiscal, lo que está relacionado al hecho de que el país, después del default, no tenía mercado externo para sus títulos públicos. La política monetaria, por su lado, pasó a ser dirigida a partir de un régimen de metas monetarias. El gobierno preanuncia antes del comienzo del año, las metas para el M2 total que el banco central perseguirá en el ańo en cuestión. ${ }^{6}$ Sin embargo, a pesar de existir metas monetarias, el pilar del régimen macroeconómico argentino es mantener el tipo de cambio real alto y competitivo, que las propias metas monetarias tienen en consideración la necesidad de comprar divisas para mantener la estrategia de flexibilidad administrada del cambio. Así, Argentina controla efectivamente el cambio y no la oferta de moneda, de modo que las metas monetarias tienen apenas un papel secundario.

Por fin, existe un último elemento que precisa ser mencionado en el contexto del tipo de cambio: las retenciones que consisten en impuestos en las exportaciones tradicionales, principalmente productos agrícolas y petróleo (Frenkel \& Rapetti, 2008: 216). De forma simplificada, las retenciones pueden ser entendidas como un impuesto fijo sobre las exportaciones, de forma que para cada unidad vendida en el exterior, el precio obtenido por el exportador en pesos estará dado por el producto del tipo de cambio por la diferencia entre el precio en moneda extranjera que el exportador obtiene menos este impuesto fijo (Amico, 2010: 17). Así, para un valor dado de tipo de cambio, se tiene que el precio obtenido por el exportador en moneda nacional con las retenciones es menor de lo que sería si este impuesto no existiese. Como los exportadores no aceptan vender internamente a precios menores de los que pueden obtener en el mercado internacional, las retenciones resultantes de disminuir el precio obtenido por los exportadores en moneda nacional, significan que una parte de los aumentos de precios externos de los commodities no será repasada para los precios en el mercado doméstico (Levy-Yeyati \& Valenzuela, 2007: 208; Amico, 2008: 46).

En la práctica, la introducción de las retenciones acabó por crear algo parecido a un sistema de tipos de cambio múltiples, lo que contribuyó "para disminuir el pass-through de las devaluaciones para los precios de bienes-salarios, pero también para captar parte de la renta obtenida por estos sectores tradicionalmente

6 En líneas generales, en este caso la autoridad monetaria establece una meta para la tasa de crecimiento del M2 en el año, la que consiste en una banda de variación interanual para el crecimiento del agregado monetario. Más exactamente, se establece un valor base como referencia para la tasa de crecimiento de M2, pero también se establece un piso y un techo para el crecimiento de este agregado monetario. 
competitivos a partir del tipo de cambio real competitivo"7 (Frenkel \& Rapetti, 2008: 216). De esta forma las retenciones funcionan no solamente como un mecanismo de combate a la inflación, sino también como una fuente de ingresos para el Tesoro.

Resumiendo, el régimen macroeconómico argentino representó una ruptura, al menos parcial, con la ortodoxia económica. Por un lado, hubo elementos de continuidad. Por ejemplo, la política fiscal estuvo basada en la búsqueda de superávits, siendo que en los primeros años del nuevo régimen macroeconómico, el país registró inclusive superávits nominales. ${ }^{8}$ Sin embargo, hubo por lo menos un importante elemento de ruptura: la política económica dejó de ser administrada a partir de la idea de que combatir la inflación es indispensable para el desarrollo. En este sentido, no hubo un ancla nominal para los precios, y el control sobre el cambio se dio mucho más en el sentido de obtener un tipo de cambio depreciado que impulsase las exportaciones que como un mecanismo para contener la inflación. De esta forma, el objetivo principal era estimular el crecimiento económico y la generación de empleos.

\section{LOS REGÍMENES MACROECONÓMICOS EN PERSPECTIVA COMPARADA}

Diferenciar los regímenes macroeconómicos seguidos por Brasil y Argentina en la última década requiere, como primer paso, retomar los hechos que los originaron. En este contexto, si es cierto que ambos regímenes fueron adoptados después de crisis cambiarias, hay que tener en cuenta también que las crisis no fueron exactamente iguales. En Brasil la crisis fue reprimida en pocos meses, y a mediados de 1999 el nuevo régimen ya estaba operando normalmente. De hecho, después de la crisis de 1998-1999, el entonces presidente brasileño Fernando Henrique Cardoso no destituyó al ministro de Hacienda, Pedro

\footnotetext{
7 Según Frenkel \& Rapetti, la tributación de los exportadores respondió por 63\% del superávit registrado por la administración federal en 2006 (Frenkel \& Rapetti, 2008: 216).

8 La búsqueda de superávits fiscales hizo que Gonçalves concluyese -inspirado en el documento Un Programa de Recuperación Popular de la Economía de la asociación argentina EDI (Economistas de Izquierda) - que el caso argentino, por lo menos al comienzo del gobierno Kirchner, no era un caso de ruptura con la ortodoxia. Según Gonçalves, existían tres caminos posibles en relación al modelo neoliberal de los ańos 1990: 1) continuidad; 2) antiliberalismo instrumental; 3) ruptura. Para él, la Argentina de Néstor Kirchner, al menos al comienzo de su gobierno, era un caso de antiliberalismo instrumental (Gonçalves, 2004: 135-140).
} 
Malan, y para el cargo de presidente del Banco Central invitó a Armínio Fraga, economista de formación ortodoxa y con experiencia en el mercado financiero.

En la Argentina, la crisis cambiaria que acabó en el abandono de la convertibilidad fue mucho más profunda e intensa que la crisis cambiaria brasileña de 1999. Debido a la opción extremista por la convertibilidad, abandonar el régimen en la Argentina fue mucho más traumático y acompañado por default, corralito y corralón. ${ }^{9}$ En el caso argentino la crisis alcanzó proporciones inmensas y la normalización de la vida económica llevó mucho más tiempo. ${ }^{10}$

Las diferencias de intensidad y duración de las crisis son asuntos claves que explican las estrategias distintas adoptadas por los dos países en los años siguientes. En Brasil la crisis más leve no afectó el prestigio de la ortodoxia económica. En este sentido, hubo un proceso de continuismo y el nuevo régimen que surgió después de 1999 no era nada más que el nuevo estado-del-arte en términos de política macroeconómica dentro de la ortodoxia, o sea, un régimen dirigido para el control de la inflación y para ganar la confianza de los mercados internacionales. Así, el nuevo régimen macroeconómico sería una versión mejorada del régimen macro anterior. ${ }^{11}$

En la Argentina, la crisis profunda que siguió al fin de la convertibilidad debilitó la posición de los defensores del modelo de los años 1990 y abrió espacio para la adopción de medidas heterodoxas. Durante la crisis de convertibilidad

9 La salida de la convertibilidad sucedió en etapas: 1) congelamiento de los depósitos y feriado bancario ( $1^{\circ}$ de diciembre de 2001); 2) default de la deuda (24 de diciembre); 3) devaluación del peso y pesificación (entre enero y marzo de 2002); 4) unificación del mercado cambiario y flexibilización del peso (febrero de 2002) (Sturzenegger \& Zettelmeyer, 2006: 200-201). El default argentino tuvo un carácter particular, pues incluyó pagos que todavía no habían vencido. Además se estima que $60 \%$ de la deuda que entró en el default pertenecía a argentinos (Sturzenegger \& Zettelmeyer, 2006: 182; 200-201). En el contexto de la pesificación, se decidió pesificar la mayor parte de las deudas contratadas en dólares a un cambio de 1 peso por dólar (más indexación al índice de precios al consumidor), mientras que los depósitos bancarios originalmente denominados en dólares fueron pesificados a un cambio de 1.40 pesos por dólar (más indexación al índice de precios al consumidor) (Frenkel \& Rapetti, 2008: 216). Para una discusión detallada de la salida de Argentina de la crisis ver Levy-Yeyati \& Valenzuela (2007).

10 La salida del sistema de convertibilidad fue un proceso traumático y fue el desenlace de una crisis que hizo caer $21 \%$ el PIB argentino en poco más de tres años, y aumentar las tasas de desempleo a 21.5\% (Frenkel \& Rapetti, 2008: 216).

11 Cuando Lula asumió la presidencia en el 2003, tampoco hubo cambios en el régimen macroeconómico. Solamente en el Segundo Gobierno de Lula (2007-2010) es posible percibir una inflexión en la política económica, aunque no hubieron cambios importantes en relación al régimen macroeconómico. 
el gobierno argentino optó por no seguir las recomendaciones del FMI (Bresser Pereira, 2007: 300 apud Lavagna, 2006). Además no se adoptó la dolarización -lo que en la práctica habría sido ahondar la opción hecha en la implantación del régimen de convertibilidad en 1991-y tampoco fue implementado el régimen de metas de inflación, opción que Brasil hizo en su crisis cambiaria de 1999.

Sin embargo es importante también reconocer que debido a la grave crisis que enfrentó, Argentina tenía en 2002 menos opciones de política económica que Brasil en 1999. En otras palabras, la crisis económica y la moratoria hicieron que los argentinos tuvieran un margen de maniobra menor que los brasileños para elegir un nuevo régimen macroeconómico. ${ }^{12}$ Por un lado, frente a un contexto de moratoria de la deuda pública, la posibilidad de endeudamiento público argentino se vio seriamente afectada, ya que la Argentina no tenía mercado en el exterior para su deuda pública. Por otro lado, la adopción de un tipo de cambio devaluado era un camino casi inevitable: después de la moratoria, las opciones de financiación externa quedaron muy limitadas, lo que a su vez inviabilizó la posibilidad de respaldar déficits en cuenta corriente (Mecon, ${ }^{13}$ 2005: 38). Obtener superávits en cuenta corriente era entonces una absoluta necesidad, ya que significaba la única forma de obtener divisas. Así, delante de la necesidad de exportar, el banco central argentino pasó, a partir del segundo semestre de 2002, a acumular reservas internacionales y a actuar para estabilizar el cambio en la paridad competitiva de 3.5 pesos por dólar (Sturzenegger \& Zettelmeyer, 2006: 201).

En este contexto, en el periodo que siguió al colapso de la convertibilidad, la depreciación del tipo de cambio y la búsqueda de superávits fiscales y en cuenta corriente era casi inescapable. Por consiguiente, la alternativa de política económica elegida en 2002 fue sensata, aunque probablemente, en un primer momento, mucho más una respuesta pragmática que una decisión estratégica.

Sucede que a partir de 2004 los capitales volvieron a la Argentina y el país pasó a registrar superávits en la cuenta de capital, de forma que obtener superávits en la cuenta corriente dejó de ser necesario. Sin embargo, viendo que el cambio depreciado permitió que las exportaciones argentinas se recuperaran

12 Según Frenkel \& Rapetti, "dado que la economía todavía estaba absorbiendo los efectos de la crisis y los mercados financieros domésticos se habían achicado tanto, el banco central decidió no seguir el régimen de metas de inflación” (Frenkel \& Rapetti, 2008: 219). Asimismo, si se hubiera optado por adoptar el régimen de metas de inflación, no había condiciones para hacerlo.

13 Mecon son las siglas para Ministerio de Economía de Argentina. 
rápidamente -lo que contribuyó para recolocar al país en el camino del crecimiento económico-, el gobierno optó por conservar la estabilidad y la competitividad del tipo de cambio, llegando incluso a adoptar controles de capitales a partir de 2005. De hecho, como destacan Frenkel \& Rapetti, el tipo de cambio depreciado pasó, gradualmente, a ser parte de la estrategia económica oficial (Frenkel \& Rapetti, 2008: 219). Así, si el cambio depreciado era una absoluta necesidad inmediatamente después de la crisis, pasó a ser efectivamente una opción estratégica del gobierno argentino. En este momento, la opción por el crecimiento económico fue una opción pensada. Esta elección fue reafirmada al sustituir al ministro de Economía Roberto Lavagna en 2005, ya que Lavagna quería en ese momento frenar el ritmo de la actividad económica debido al agravamiento de la inflación.

Después de estas primeras observaciones que aclaran por qué estos países optaron por estrategias diferentes, toca ahora discutir los méritos y las deficiencias de ambos regímenes, lo que requiere tener en consideración los resultados del desempeño económico. En este contexto, desde el punto de vista de los resultados obtenidos por los dos regímenes macroeconómicos, hay que considerar los objetivos que estos regímenes pretendían alcanzar. En Brasil hay, claramente, una declarada tendencia de combate a la inflación. En la Argentina, por otro lado, la prioridad fue el crecimiento económico a través del estímulo de las exportaciones y la recuperación del mercado nacional.

En el caso brasileño los resultados muestran que, desde el punto de vista de las metas establecidas, el régimen macroeconómico tuvo éxito. La inflación -objetivo de la política monetaria- estuvo dentro de la meta en la mayor parte del periodo. Es verdad que hubo momentos de turbulencia entre 2001 y 2004, y que en los años 2003 y 2004 fue necesario ajustar las metas ${ }^{14}$ (Céspedes et al., 2005: 21). Sin embargo, el ajuste en 2003-2004 no es suficiente para declarar que el régimen de metas fracasó en el control de la tasa de inflación. ${ }^{15}$ Como puede verse en la Gráfica 1, las tasas de inflación estuvieron en la mayor parte del tiempo dentro de los intervalos máximos y mínimos de las metas inflacionarias, que corresponden al área sombreada. ${ }^{16}$

14 En 2003, el intervalo de la meta fue alterado de $1.25-6.25 \%$ para $1.5-6.5 \%$. En 2004, el intervalo fue alterado de $1.25-6.25 \%$ para 3-8\%.

15 La crítica principal al régimen de metas de inflación brasileño es que la inflación haya sido controlada usando una de las tasas de interés más altas del mundo. Para detalles, ver Modenesi \& Werneck Vianna (2010).

16 El área fue construida considerando apenas las metas ajustadas de los años 2003 y 2004. 
Gráfica 1. Tasas de inflación en Brasil (1999-2010)

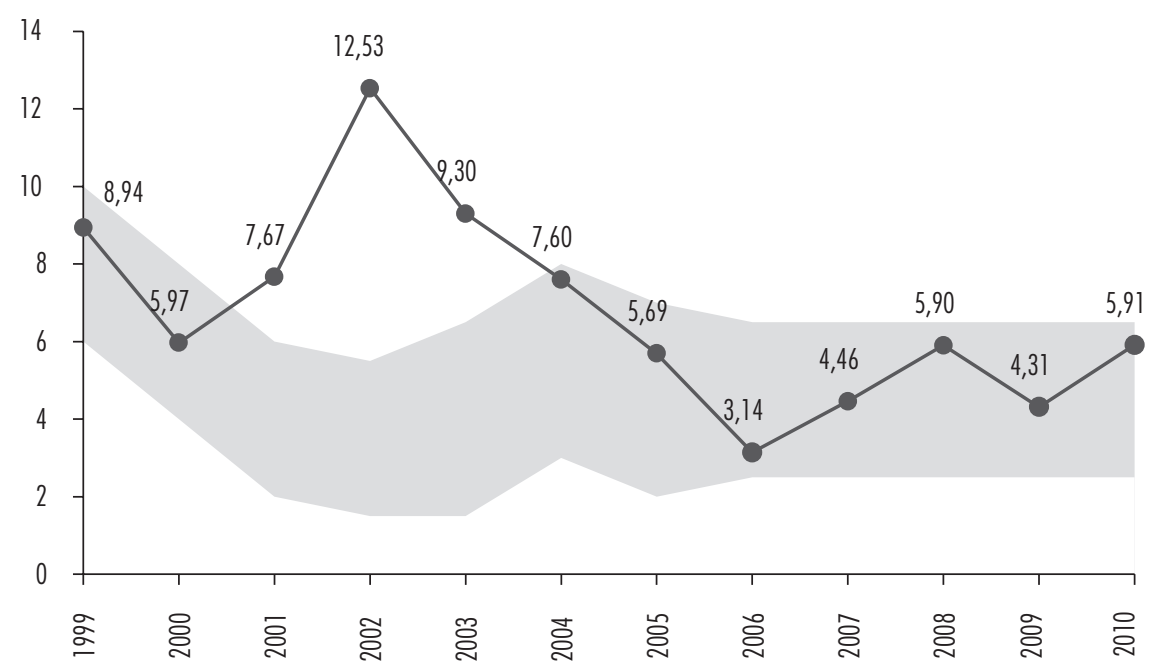

Fuente: Banco Central de Brasil.

En relación con las cuentas públicas, también se cumplieron las metas establecidas. A partir de 1999 el país pasó a registrar sistemáticamente superávits primarios sobre el PIB, siendo que entre 2001-2008 el valor de esta variable estuvo siempre igual o arriba de 4\%, excepto solamente en el año 2006 cuando el superávit primario sobre el piB fue de 3.9\%. Se observa también una caída del déficit nominal/PIв de $5.8 \%$ en 1999 para $1.5 \%$ en 2008, mientras que la Deuda Líquida sobre el Superávit Primario/pib cayó de 44.5\% en 1999 para 36\% en 2008 (Modenesi \& Werneck Vianna, 2010: 115).

Finalmente, la economía creció un promedio de $3.4 \%$ en el periodo 1999 2010 y $4.4 \%$ en el periodo 2004-2010, habiendo alcanzado tasas superiores a $5 \%$ en cuatro años $(2004,2007,2008$ y 2010). Si estas tasas no llegan a ser importantes, pueden ser consideradas por lo menos razonables, todavía más si tenemos en cuenta que en las décadas de 1980 y 1990 las tasas de crecimiento fueron muy pequeñas.

Frente a este cuadro, el principal problema del régimen macroeconómico brasileño es la tendencia a la apreciación del tipo de cambio. Según datos del Banco Central de Brasil, el tipo de cambio real brasileño empezó en 2003 un proceso de apreciación y se quedó en diciembre de 2010 en un valor equivalente a $70 \%$ del tipo de junio de 1994 , un valor inferior al tipo vigente en diciembre de 1998, momento en que era opinión casi generalizada la de que había un problema de atraso cambiario en Brasil. 
La apreciación cambiaria puede representar un obstáculo serio para el futuro, ya que tiende a provocar el aumento del déficit en cuenta corriente y, lo que es mucho peor, puede llevar al país a un proceso de desindustrialización y/o reprimarización de la pauta de las exportaciones. ${ }^{17}$ Estos elementos fragilizan la economía brasileńa, pues a corto plazo los altos precios internacionales de los commodities ayudan a respaldar mínimamente los saldos comerciales. Sin embargo el aumento de la creciente dependencia de los productos primarios es preocupante, pues el país queda más expuesto a las oscilaciones de los precios internacionales de estas mercaderías.

Tomando en cuenta que China tiene un peso muy importante en el aumento reciente de los commodities y que esta creciente participación se debe al rápido proceso de industrialización y crecimiento por el cual su economía pasa en este momento, es bastante posible que en el mediano plazo, como comenzará a crecer en tasas menores, los precios de los commodities se ajustarán en niveles más bajos. Es el caso, por ejemplo, del precio de los metales. Según el Fondo Monetario Internacional (2006), "los modelos históricos sugieren que el consumo de metales crece típicamente en conjunto con la renta per cápita hasta llegar a un nivel de $\$ 15,000-\$ 20,000$ (en términos de paridad del poder de compra) a medida que el país pasa por un proceso de industrialización y montaje de infraestructura" (FMI, 2006: 6). Como los niveles de renta per cápita de China todavía no se encuentran en este punto, deberá haber algunos años, según la lógica, de precios de metales en alza. Sin embargo, es un hecho que este ciclo de alza no es un proceso perenne.

En este contexto, el problema es que frente a un tipo de cambio apreciado, los precios elevados de los commodities mínimamente garantizan el lucro de las exportaciones de productos primarios. Sin embargo, no sucede lo mismo con los productos industriales, que precisan un tipo de cambio más deprecia$\mathrm{do}^{18}$ (Bresser Pereira, 2007: 121). De esta forma, los sectores industriales sufren

17 Es importante distinguir desindustrialización de reprimarización de la pauta de exportaciones. La desindustrialización -tal como está redefinida por Tregenna (2009)-se caracteriza por una situación en que hay tanto una reducción de empleo industrial en relación al empleo total cuanto una caída del valor agregado por la industria al PIB. La reprimarización de la pauta de exportaciones, a su vez, se caracteriza por el aumento de la participación en la pauta de las exportaciones de productos primarios y fabricados de bajo valor agregado y/o bajo contenido tecnológico (Oreiro \& Feijó, 2010: 221-222).

18 Bresser Pereira habla de un tipo de cambio de equilibrio industrial que sería "la tasa que viabiliza industrias de bienes comercializables utilizando la mejor tecnología mundial” (Bresser Pereira, 2010: 69). 
doblemente con el cambio apreciado: por un lado, estos sectores tienen un ingreso menor en reales por producto exportado y, por otro lado, tienen que enfrentar en el mercado nacional la competencia de productos extranjeros que llegan con precios más competitivos. Con esto, el riesgo de este proceso es que la industria brasileña no resista a esta competencia más pesada y el país sufra una desindustrialización. Si esto llega a suceder, Brasil quedará muy vulnerable cuando acabe el ciclo de alta de los commodities.

$\mathrm{Al}$ analizar la existencia de estos problemas hoy en Brasil, se ve que hay fuertes evidencias de reprimarización de la pauta exportadora. Un ejemplo es el hecho de que la participación relativa de las exportaciones brasileñas de básicos, según datos del anuario estadístico de 2009 de la CEPAL, ha aumentado de 46\% en 2001 a $55.4 \%$ en 2009.

En relación a la desindustrialización, no está claro si este proceso ya está caminando en Brasil. ${ }^{19}$ Con todo, hay algunas evidencias de las dificultades por las que pasa hoy la industria brasileña. Una evidencia se encuentra en los datos de coeficiente de penetración de las importaciones ${ }^{20}$ en el periodo 2004-2010 (Cuadro 1). Estadísticas del Ipeadata muestran un salto en el coeficiente de penetración de las importaciones en la industria de transformación de $11.1 \%$ en el cuarto trimestre de 2004 para $19 \%$ en el cuarto trimestre de 2010 . Sectorialmente, este valor casi triplicó en el caso de la industria textil y casi duplicó en el caso de vehículos en ese periodo. ${ }^{21}$ En el caso de máquinas y equipos, hubo un aumento de casi $65 \%$ también en ese periodo.

De cualquier forma, aunque hasta el momento no hay acuerdo sobre los efectos de la apreciación cambiaria sobre la industria, existe una cierta convergencia entre los autores de que si se mantiene la tendencia actual, los problemas de desindustrialización y reprimarización de la pauta de las exportaciones surgirán en el futuro. ${ }^{22}$

19 Hay trabajos -como Oreiro \& Feijó- que afirman que hubo desindustrialización en Brasil en los últimos años, en vista de la presencia simultánea de pérdida de participación de la industria de transformación en el PIB y además el aumento del déficit comercial de la industria (Oreiro \& Feijó, 2010: 229-230).

20 Es el coeficiente de la participación de las importaciones en el consumo aparente.

21 El dato de vehículos incluye vehículos con motor, remolques y carrocerías.

22 El riesgo de reprimarización de la pauta de las exportaciones, y en particular de desindustrialización, debe ser señalado por la importancia que la industria adquirió en la economía del país. A lo largo del siglo xx Brasil realizó un gran esfuerzo para desarrollar la industria, esfuerzo que permitió que el país tuviese al comienzo de los años 1980 una base industrial integrada y bastante diversificada (Versiani \& Suzigan, 1990: 21). 
Frente a este contexto, parece entonces claro que el proceso de apreciación cambiaria tiene que ser revertido. El problema, en el caso del debate más amplio del régimen macroeconómico brasileño, es que la apreciación cambiaria ha sido un factor importante para conseguir las metas de inflación. En la mitad de la década actual, comenzó un fuerte ciclo de aumento de precio de los commodities, lo que en la práctica significó un choque de oferta que, sin intervención, iría a provocar un aumento de las tasas de inflación en Brasil. Sin embargo, la apreciación cambiaria en el periodo amortiguó este choque e impidió que se transmitiera para los precios internos (Serrano \& Ferreira, 2010: 53). La Gráfica 2 muestra este blindaje logrado por la apreciación cambiaria en el periodo. En este caso puede verse que el aumento del índice de los commodities CRB en reales quedó abajo del aumento en dólares, lo que significa que solamente una parte del aumento de los precios de los commodities se transmitió para la economía brasileña.

Gráfica 2. Índice CBR (dólares y reales)

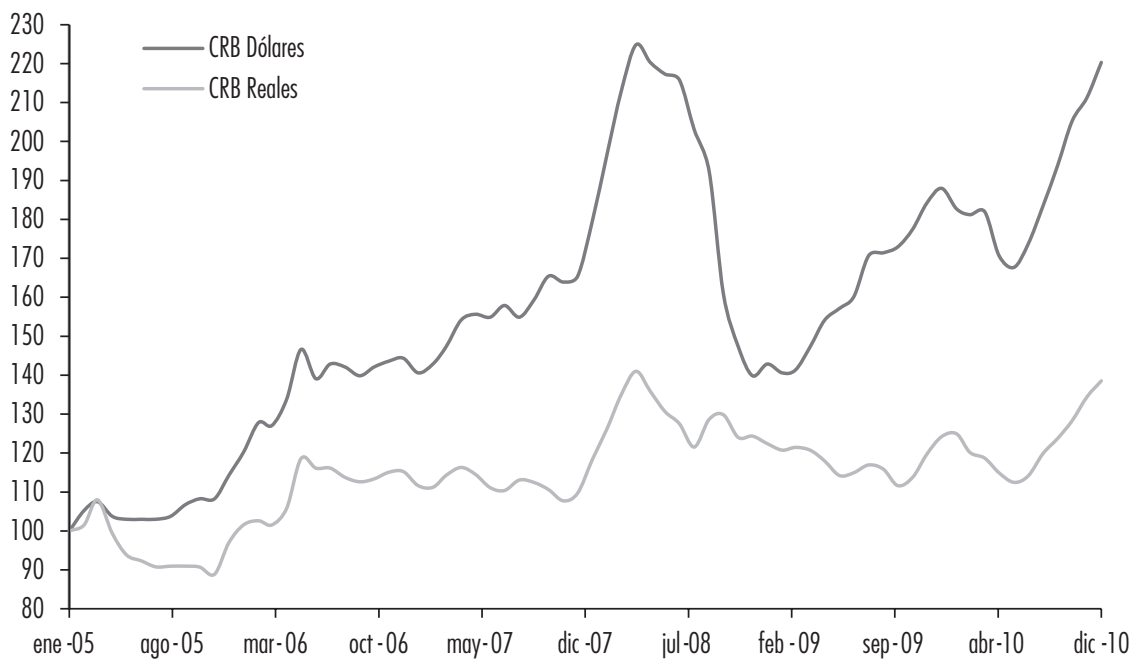

Fuente: Elaboración propia a partir de datos del Banco Central de Brasil y UNCTAD.

La importancia de la apreciación del tipo de cambio en el control de la inflación en Brasil no se restringe, sin embargo, al periodo reciente de alza del precio internacional de los commodities. Según Barbosa-Filho (2009), exceptuando el año 1999, las metas de inflación en el periodo 1999-2006 sólo fueron alcanzadas en los años en que el tipo de cambio se apreció en términos 
nominales, o sea, en 2000, 2004, 2005 y 2006 (Barbosa-Filho, 2009: 142). Para este autor esta relación se debió, entre otras cosas, al hecho de que, después de las privatizaciones de los años 1990, los precios de algunos servicios de utilidad pública pasaron a seguir índices de precios muy influenciados por el tipo de cambio $^{23}$ (Barbosa-Filho, 2009: 140).

En este contexto queda claro que la apreciación cambiaria está siendo, efectivamente, un mecanismo importante para alcanzar las metas de inflación desde que el régimen fue adoptado (Serrano, 2010: 65). En este caso el problema es que en tanto esta apreciación cambiaria no sea intención del Banco Central, el cambio más apreciado es un by-product de la política monetaria ejecutada para cumplir las metas inflacionarias: al aumentar los intereses, la autoridad monetaria acaba aumentando el diferencial de la tasa doméstica con relación a las tasas externas, atrayendo capitales extranjeros y apreciando el tipo de cambio.

El problema es que, como vimos anteriormente, la tendencia de apreciación del tipo de cambio trae riesgos importantes en lo concerniente a la estructura económica. Así, parece existir un impase: si, por un lado, la apreciación cambiaria trae los riesgos de la desindustrialización, por otro, permitir un tipo de cambio más devaluado tendrá consecuencias sobre la inflación doméstica. Esta conjunción de factores parece señalar un impase en términos de política económica. ${ }^{24}$

Pasando ahora al caso argentino, puede observarse que el régimen macroeconómico del vecino país también presentó resultados favorables en relación a las metas que quiere alcanzar. Desde el punto de vista del crecimiento económico, meta primordial del gobierno, la economía creció a una tasa promedio impresionante de $8.5 \%$ al año en el periodo 2003-2008. En función de la crisis internacional, la economía estuvo paralizada en 2009, recuperándose rápidamente en 2010. Además de esto, durante este ciclo de crecimiento la industria argentina volvió a desarrollarse. Como el sector industrial sufrió muchas pérdidas durante el periodo de la crisis de convertibilidad, hoy se habla de que Argentina está en un proceso de reindustrialización. A pesar de no haber habido cambios importantes en el perfil de la industria, ${ }^{25}$ hubo señales de recuperación en relación a la fase de convertibilidad, como por ejemplo el hecho de que

\footnotetext{
23 Otra razón es que los bienes negociables son, por su naturaleza, influenciados por el comportamiento del tipo de cambio.

24 Oreiro (2011) también llegó a la misma conclusión.

25 Para detalles, ver Azpiazu \& Schorr (2010).
} 
"las exportaciones de origen industrial aumentaran en quantum a una tasa promedio de 12\% entre 2002 y 2007 " (Amico, 2008: 35).

Además, la Argentina también ha venido acumulando reservas internacionales casi sistemáticamente desde 2003. Según los datos del Banco Central de Brasil, las reservas argentinas llegaban a un total de 9 mil millones de dólares en julio 2002 y en diciembre 2010 ascendieron a casi 50 mil millones de dólares.

Sin embargo, así como en el caso brasileño, el régimen argentino también ha generado efectos colaterales indeseables. En la Argentina, no obstante, el principal problema resultante de la adopción del régimen actual está en el resurgimiento de la inflación. $\mathrm{Al}$ aceptar este reciente recrudecimiento de la inflación en la Argentina, se hace necesario examinar el desempeño de la estrategia económica utilizada en el país.

El tipo de cambio estable y competitivo de los primeros años del nuevo régimen permitió una respuesta rápida de las exportaciones, la que junto con el boom en el mercado internacional de los commodities, proporcionó superávits en la balanza comercial y también contribuyó para obtener superávits en cuenta corriente, los cuales fueron, en el periodo 2003-2006, del orden de 3\% del PIB. Al mismo tiempo, la disminución del pago de intereses, el aumento de la recaudación tributaria (en función del retorno del crecimiento económico) y la tasación sobre las exportaciones tradicionales, contribuyeron para obtener, en el promedio del periodo 2003-2006, superávits primarios equivalentes a $4 \%$ del рів (Cunha \& Ferrari, 2009: 9-11 apud FMI, 2005). Se creó entonces una combinación de superávits gemelos (Cunha \& Ferrari, 2009: 19, Cárdenas et al., 2010: 44).

Inicialmente la maxidevaluación cambiaria de 2002 no provocó aumentos de salarios: frente a una tasa de desempleo de $22 \%$, las demandas salariales fueron prorrogadas, lo que se tradujo en el mantenimiento del salario nominal y disminución del salario real (Levy-Yeyati \& Valenzuela, 2007: 207). Así, en esta primera fase del nuevo régimen, fue posible tener tasas de crecimiento altas y aumento de los niveles de empleo, al mismo tiempo en que las tasas de inflación permanecían en niveles aceptables (Curia, 2007: 127-28).

El problema inflacionario comenzó cuando la economía pasó a acercarse a los niveles de pre-crisis. ${ }^{26}$ En este momento, con la economía creciendo y las tasas de desempleo cayendo, los trabajadores comenzaron a pedir reajustes salariales para reponer las pérdidas reales sufridas después de la maxidevaluación de

26 El análisis de la inflación actual en Argentina está basado en gran medida en Amico (2008; 2010). 
2002, de forma que los salarios reales empezaron a aumentar. Así los salarios reales -calculados a partir del índice de precios al consumidor oficial del Instituto Nacional de Estadística y Censos (INDEC) - iniciaron un movimiento ascendente a partir de 2003 y, a partir de 2007, comenzaron a registrar aumentos reales en relación a los niveles anteriores a la crisis ${ }^{27}$ (Fabris et al., 2009: 23).

Además hubo también, en este mismo periodo, otro refuerzo de este proceso: el aumento de precio de los commodities en el mercado internacional. El aumento del precio internacional de los commodities agrícolas - productos de exportación de la Argentina- presionó los precios en el mercado interno, pues los productores no aceptan vender internamente a precios más bajos de los que pueden obtener en el mercado internacional. Además, la mayor parte de la pauta de las exportaciones argentinas está formada por bienes que tienen un peso grande en la canasta de consumo popular, lo que afecta el poder adquisitivo de los trabajadores y les estimula a intentar reponer estas pérdidas (Amico, 2008: 46; Levy-Yeyati \& Valenzuela, 2007: 208). Frente a las presiones por aumentos salariales, los empresarios intentan, por otro lado, aumentar los precios para defender la participación de las ganancias en el ingreso, presionando para arriba las tasas de inflación. Así, como sugiere Amico (2008; 2010), la actual inflación argentina parece vinculada con el choque de costos -asociado al ajuste cambiario y la alza de los commodities - y con el conflicto distributivo resultante (Amico, 2008: 43, 46; Amico, 2010: 14, 20).

El problema es que, como la estrategia argentina está basada en un tipo de cambio real estable y competitivo, la apreciación cambiaria no puede ser usada como amortiguador para las presiones inflacionarias provenientes del ciclo de alza de los commodities. Entonces, si la apreciación cambiaria permitió al Brasil atenuar el choque de los commodities, Argentina sufrió los impactos de este choque. Esta afirmación es confirmada por la Gráfica 3, que presenta la evolución del índice CRB en reales y en pesos argentinos, mostrando así el impacto mucho mayor del choque de commodities sobre los precios en la Argentina. ${ }^{28}$

27 Deflacionando con un índice de precios alternativo - como por ejemplo, el IPC 7 provincias-, las ganancias reales de los trabajadores a partir de 2007 son, no obstante, mucho menores de las obtenidas a partir del índice de INDEC. Esta diferencia sucede debido al hecho de que, desde 2007, se sospecha que el INDEC haya calculado índices que subestiman la inflación verdadera.

28 Según Amico (2010), la política cambiaria argentina amplió el choque de costos. Para él, el tipo de cambio competitivo y la estructura de la canasta exportadora explican las diferencias de niveles de inflación de la Argentina en comparación no solamente con Brasil sino también con Chile, Colombia y México (Amico, 2010: 13). 
Gráfica 3. Índice CRB (pesos y reales)

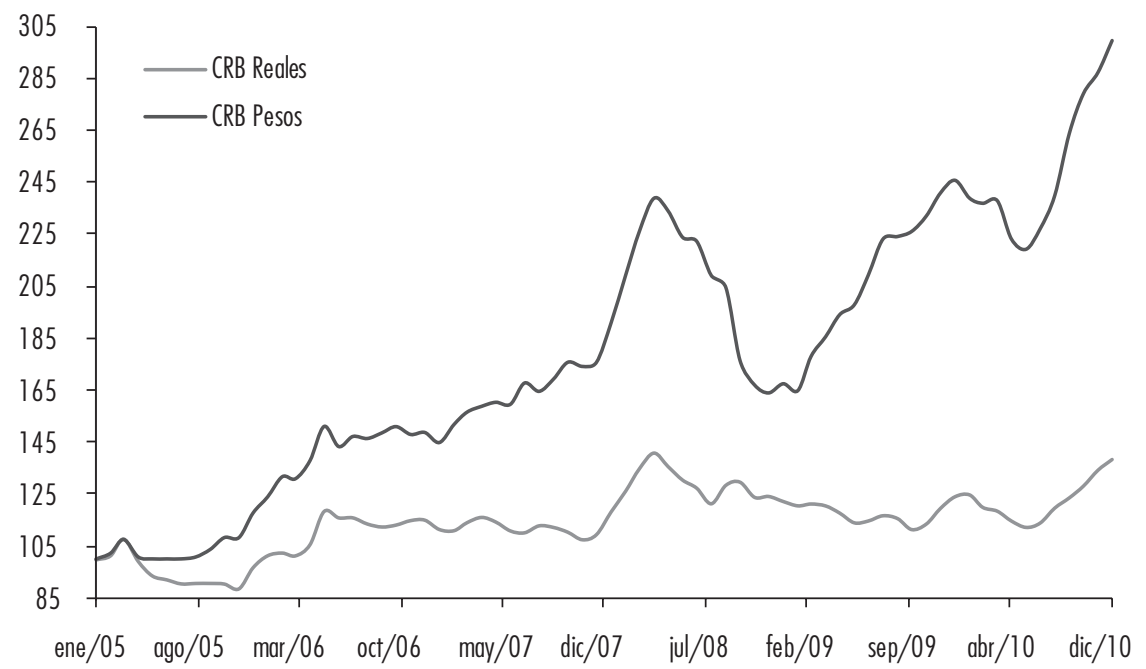

Fuente: Elaboración propia a partir de datos del Banco Central de Brasil, Banco Central de la República Argentina y unctad.

La Gráfica 4 complementa la información de la Gráfica anterior al mostrar la evolución diferente de los índices de precio al consumidor de Brasil y Argentina (IPCA e INDEC) a partir de 2005. ${ }^{29}$

Sin poder apelar para el cambio, el gobierno argentino buscó hacer acuerdos de congelamiento de precios, con énfasis en la estabilización de los precios de productos de la canasta básica (Cunha \& Ferrari, 2009: 19). Además se usó como instrumento de combate a la inflación la antigua política de las retenciones (tasas sobre las exportaciones), las que fueron utilizadas para atenuar el problema inflacionario al proteger - por lo menos en forma parcial- los salarios de los efectos de la devaluación. El problema es que, aunque ciertamente hayan contribuido a impedir un aumento más grande aun de los precios domésticos, congelamientos y retenciones son instrumentos que tienen limitaciones en el combate a la inflación. Los congelamientos, por ejemplo, tienen que ser temporales, pues pueden generar especulación y el surgimiento del mercado

29 Si tomamos como referencia algún índice alternativo de precios -como IPC 7 provincias calculado por el Centro de Estudios para el Desarrollo Argentino (Cenda)-, la diferencia entre los precios al consumidor en Argentina y Brasil será aún mayor de lo expuesto en la Gráfica 4. 
negro. En el caso de las retenciones, están los problemas políticos que crean con el sector agrario, como la crisis política que hubo en el año 2008, cuando el gobierno de la presidenta Kirchner intentó instituir retenciones móviles. ${ }^{30}$

Gráfica 4. Índice de Precios al Consumidor. Argentina y Brasil

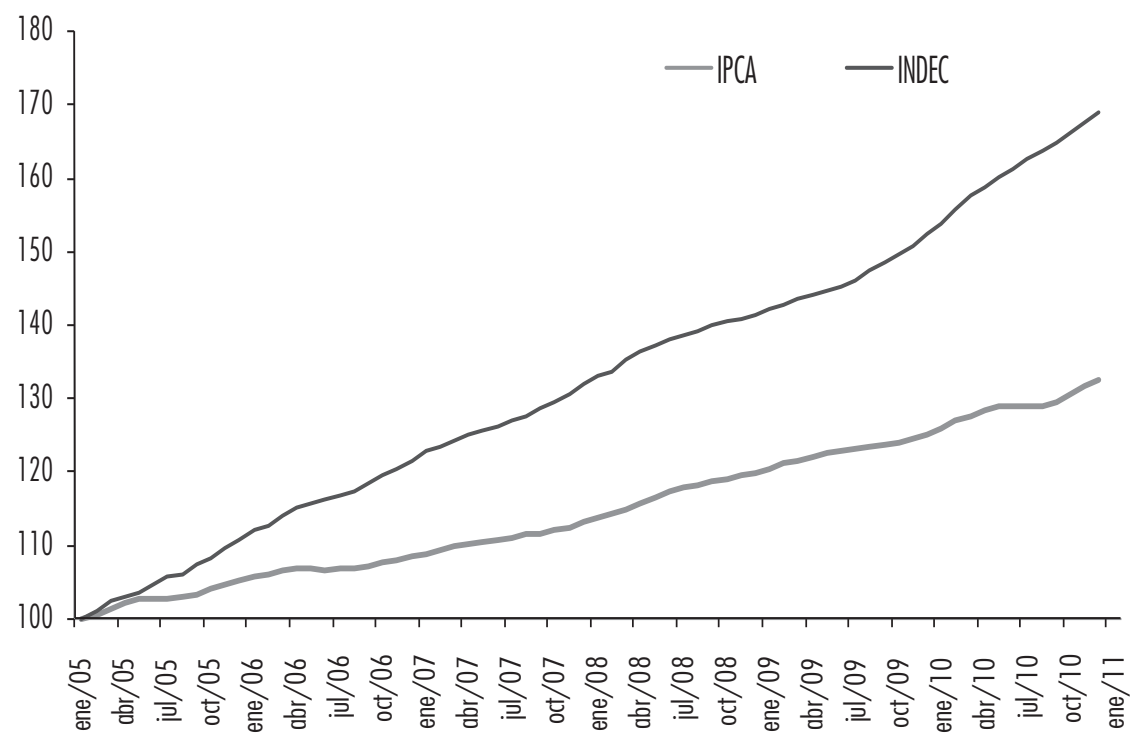

Fuente: Elaboración propia a partir de datos del Banco Central de Brasil y Mecon.

Frente a este contexto, el problema es que el retorno de las presiones inflacionarias crea una serie de dificultades para la economía argentina, pues aprecia el tipo de cambio real, lo que tiende a provocar la caída de las exportaciones y el

30 Las retenciones móviles consisten en impuestos que acompañan la dinámica de los precios internacionales de los commodities agropecuarios, de forma que la diferencia entre el precio internacional de estos commodities y la tasa cobrada por unidad exportada se vuelve constante. Suponiendo un tipo de cambio fijo, los aumentos en los precios internacionales de los commodities agropecuarios no se transmiten, con retenciones móviles, para los precios domésticos de estos bienes. Así, las retenciones móviles podrían blindar la economía argentina de las presiones de inflación de los commodities, a diferencia de las retenciones tradicionales que, por ser fijas, afectan sólo de forma parcial la tendencia de inflación (Amico, 2010: 3; 18-19). 
deterioro de la balanza comercial a lo largo del tiempo. Sucede que desde 2007 hay dudas en la Argentina sobre la confiabilidad de los índices de precios presentados por el INDEC, ${ }^{31}$ de manera que si las estimativas de inflación alternativas al INDEC están correctas, el tipo de cambio real argentino ya se está apreciando. En el año 2011 existía un cierto consenso acerca de que Argentina no sufría de atraso cambiario, pero se creía que mantenidas las actuales tasas de inflación, este problema surgiría en breve, lo que probablemente afectaría de forma negativa los saldos comerciales argentinos.

El deterioro del cuadro de las cuentas externas no es diferente de lo que sucede en las cuentas públicas. Según Cárdenas et al., retirando los aumentos de ingresos derivados de la nacionalización de los fondos de pensión y de los ingresos cuasifiscales extraordinarios transferidos del Banco Central en 2010, el superávit primario/PIB cayó 5 puntos porcentuales en los últimos tres años, siendo que desde 2009 el país estaría registrando déficits primarios. A pesar de este déficit ser administrable, los autores destacan la velocidad del deterioro del cuadro fiscal (Cárdenas et al., 2010: 44-5). Así, en la Argentina actual el escenario de superávits gemelos parece ya ser cosa del pasado.

Frente a esta situación, desde el punto de vista del régimen macroeconómico argentino, el gobierno tendría, primeramente, que promover una devaluación cambiaria. Sin embargo, como la Argentina hoy crece bien y tiene-como parece ser el caso- una inflación alrededor de 20-30\% al año, una devaluación tiende a causar más presiones inflacionarias. Los aumentos de precios y salarios pueden hacer que la devaluación nominal tenga poco o ningún efecto real, lo que exigirá una nueva devaluación, disparando así una espiral cambio-salarios y

31 Según Damill \& Frenkel (2009), el gobierno argentino pretendía mantener la tasa de inflación en hasta una cifra. Como en 2007 el gobierno tuvo dificultad en alcanzar esta meta, resolvió controlar el índice, en lugar de controlar efectivamente la inflación. Así, hubo alteraciones en el cálculo del IPC del INDEC, y éste empezó a variar mucho menos que los índices de inflación calculados por asesorías privadas y por los institutos de estadística de las provincias (Damill \& Frenkel, 2009: 16-19). Con esto se tiene que, entre otras cosas, las pérdidas de los salarios reales son subestimadas, lo que induce, al menos a corto plazo, reajustes salariales menores a la inflación efectiva. Además, el índice de inflación del INDEC es base de cálculo del coeficiente que se "usa para el ajuste de deudas, principalmente de buena parte de las obligaciones en pesos emitidas por el gobierno nacional durante la reestructuración hecha en 2005" (Damill \& Frenkel, 2009: 20). De esta forma, como observan Cárdenas et al., la subestimación del índice de inflación de INDEC reduce el servicio de la deuda y ha llevado a algunos acreedores -aunque no a las agencias de riesgo- a ver este hecho como un default implícito (Cárdenas et al., 2010: 47). 
salarios-precios, en la cual trabajadores y empresarios tratan de defender sus ingresos. ${ }^{32}$ El resultado puede ser un alza repentina del proceso inflacionario, de forma que la devaluación nominal ni siquiera ocasionará una devaluación real del tipo de cambio. Además, frente a una inflación creciente y ya del orden de 20-30\%, existe siempre el riesgo de una indexación informal que si sucede, hará más difíciles los esfuerzos antiinflacionarios en el futuro. Como los sindicatos están aliados con el gobierno, sus reclamos por reajustes no son generalmente exagerados, lo que ha ayudado hoy a impedir un aumento mayor de la inflación. Sin embargo, no está claro cuál será la actitud de los sindicatos si la inflación se acelera en el futuro.

\section{BALANCE GENERAL HASTA EL AÑO 2010 Y DESAFÍOS PARA EL FUTURO}

Al principio los resultados presentados por los dos regímenes macroeconómicos muestran un éxito razonable cuando se tienen en cuenta las opciones hechas por los dos países. En el Brasil, donde la estabilidad de precios fue la prioridad, las tasas de inflación se mantuvieron bajo control. En la Argentina, donde la prioridad fue el crecimiento económico y la recuperación industrial, las metas también fueron alcanzadas. Naturalmente, en los dos casos, hubo efectos colaterales negativos, pero desde el punto de vista específico de las prioridades establecidas, los dos regímenes macroeconómicos tuvieron éxito. ${ }^{33}$

En relación con la continuidad de los dos regímenes, hay desafíos enfrente. En Brasil, el régimen macro puede ser mantenido sin mayores dificultades en corto plazo. El problema son los efectos que mantener el régimen actual tendrá

$32 \mathrm{Al}$ analizar la experiencia alemana de hiperinflación en los años 1920, Joan Robinson observó este tipo de proceso. Según ella, la depreciación cambiaria aumentaba el costo de vida y provocaba reclamos salariales. Los salarios más altos, a su vez, aumentaban los costos y los ingresos domésticos, eliminando con esto los efectos de la depreciación de estimular las exportaciones y desestimular las importaciones. De esta forma cada devaluación llevaba a aumentos salariales, lo que hacía caer el tipo de cambio induciendo nuevas devaluaciones cambiarias que acabarían resultando en nuevos aumentos salariales (Robinson, 1938: 510). Para una discusión de estos mecanismos de propagación de la inflación ver Bastos (2001) y Câmara \& Vernengo (2001).

33 Como este análisis no incluye las políticas industriales y estas políticas afectan variables como crecimiento e inflación, debe ser resaltado que los resultados no pueden ser atribuidos exclusivamente a los regímenes macroeconómicos. 
sobre la estructura industrial y la pauta de las exportaciones a medio y largo plazos. Así, existe todo un conjunto de medidas que deben ser tomadas para detener la continuidad de la apreciación real del tipo de cambio, así como formas alternativas de combate a la inflación que permitan no tener que recurrir más a la apreciación nominal del cambio. En este sentido existen algunas posibilidades, como por ejemplo la propuesta de Serrano \& Summa (2011) de "disminuir temporalmente los impuestos a las importaciones de bienes básicos cuyos precios son muy volátiles y que estén visiblemente creciendo mucho", así como "colocar impuestos a las exportaciones de bienes básicos cuando su precio en dólares aumenta mucho en un corto periodo de tiempo, para impedir que estos aumentos sean repasados para los productos domésticos" (Serrano \& Summa, 2011: 33). Otra posibilidad -planteada por Modenesi et al. (2010) y Nakano (2011) - es aumentar la potencia de la política monetaria a través de la desindexación de los activos financieros, ya que éstos siguen indexados a la tasa diaria de interés. ${ }^{34}$ Finalmente, en relación a la apreciación nominal del cambio, una posibilidad es el uso de controles de capitales para atenuar el efecto apreciador que la gran entrada de recursos está generando. ${ }^{35}$ En este ítem, el gobierno ha tomado algunas iniciativas. ${ }^{36}$

En la Argentina la continuidad del régimen macro actual es mucho más complicada. La inflación creciente está provocando la apreciación real del tipo de cambio, lo que genera a propósito una situación curiosa: a pesar de las enormes diferencias entre los regímenes macro de Argentina y Brasil, los dos países están enfrentando hoy, aunque por razones distintas y en intensidades diferentes, el mismo problema de apreciación real del tipo de cambio. En la Argentina el problema de apreciación cambiaria es que, para conservar el régimen macro actual, sería necesaria una nueva devaluación nominal del cambio. Como esta opción no es aconsejable debido a sus efectos inflacionarios, la salida es combatir la inflación que existe hoy en el país. El problema es que todas las opciones en este sentido enfrentarán problemas políticos. La adopción

\footnotetext{
34 Según los autores, ésta sería una reminiscencia innecesaria de los tiempos de inflación alta.

35 Gala (2010) presenta una serie de propuestas en este sentido.

36 Hubo un conjunto de medidas que fueron tomadas desde 2010. En el mercado de renta fija hubo, en octubre de 2010, un aumento del Impuesto sobre Operaciones Financieras (IOF) incidente sobre los ingresos de recursos extranjeros para renta fija de títulos públicos y privados de $4 \%$ para $6 \%$, medida que fue parcialmente flexibilizada al principio de diciembre de aquel año. En el mercado futuro, por ejemplo, fue introducido durante el 2011 el cobro de IOF de $6 \%$ para algunas categorías de préstamos de bancos y empresas brasileñas en el exterior.
} 
de retenciones flexibles encontrará fuerte resistencia del sector rural, como ya sucedió en 2008. Por otro lado, adoptar políticas de contracción y permitir la apreciación nominal del peso traerá insatisfacción al sector industrial, que no cuenta con los mismos instrumentos de ayuda gubernamental de sus pares brasileños ${ }^{37}$ y que, además, ya reclama hoy que el tipo de cambio real argentino está muy apreciado. Finalmente, no es posible tampoco contener la inflación a través del control de salarios, pues además de los efectos regresivos de este tipo de política, los sindicatos son importantes aliados del gobierno.

Así, como todas las soluciones enfrentan problemas políticos, la situación actual recuerda los análisis clásicos de Canitrot ([1975] 1991) y Mallon \& Sourrouille (1973), que describían a la Argentina como una sociedad conflicti$v a^{38}$ y señalaban las dificultades para montar coaliciones e implantar políticas económicas en el país. ${ }^{39}$

En síntesis, en este contexto de conflictividad, la solución tal vez pase por algún arreglo en términos de políticas de ingresos. ${ }^{40}$ De todos modos, cualquiera que sea la solución propuesta, su éxito dependerá naturalmente de alguna forma de pacto político.

\section{OBSERVACIONES FINALES}

En síntesis, Brasil y Argentina recorrieron caminos muy diferentes después de las crisis cambiarias, y delante de los objetivos propuestos por los respectivos

37 No existe en la Argentina un banco de desarrollo como el BNDEs brasileño.

38 En este caso, el problema es que la sociedad argentina es tradicionalmente más conflictiva que las otras y, en particular, más conflictiva que la brasileña.

39 En el contexto de una sociedad conflictiva como la argentina, Mallon \& Sorrouille (1973) afirmaban que "es necesario que las macropolíticas... sean elaboradas en forma tal que permitan movilizar el apoyo de una coalición de fuerzas suficientemente poderosa como para sostener al gobierno en el poder" (Mallon \& Sourrouille, 1973: 270-1). Por otro lado, Canitrot (1991) afirmaba que en las fases de recesión surgía una alianza populista entre trabajadores y la burguesía industrial orientada al crecimiento económico, que duraba hasta que la economía alcanzaba el pleno empleo. En este momento los intereses comenzaban a entrar en conflicto, y este conflicto era acentuado por la explosión de la inflación. El populismo iría a acabar con la burguesía industrial, confirmando una nueva alianza con la burguesía agraria basada en un programa de orden y recesión (Canitrot, 1991: 28-29).

40 La adopción de política de ingresos es una solución sugerida por De Paula (2011) y Amico (2010). 
regímenes macroeconómicos, por lo menos obtuvieron resultados razonables considerando las prioridades establecidas. En Brasil las tasas de inflación permanecieron en niveles relativamente bajos, mientras que en la Argentina las tasas de crecimiento fueron altas y la industria se recuperó.

Sin embargo, adoptar estos regímenes trajo también efectos negativos. En Brasil, el foco de inflación llevó a la apreciación cambiaria que blindó a la economía brasileńa de los impactos inflacionarios del ciclo de alza de los commodities, pero por otro lado ha provocado un proceso de reprimarización de la pauta exportadora, exponiendo al país al riesgo de desindustrialización. En la Argentina, la estrategia de tipo de cambio estable y competitivo impidió el uso de la apreciación cambiaria como mecanismo de blindaje contra la inflación de commodities. Como consecuencia de este choque de alza de los commodities y de las presiones por aumentos salariales, las tasas de inflación se elevaron y hoy han provocado también la apreciación real del tipo de cambio argentino, amenazando el principal pilar del régimen macroeconómico argentino.

En este contexto, estos problemas consisten hoy en desafíos para brasileños $y$ argentinos, de modo que se hace necesario efectuar cambios en el curso actual de los regímenes macroeconómicos, pues si no se hacen, las dos economías enfrentarán dificultades más serias en el futuro. En resumen, el éxito relativo de la primera década del siglo XXI no puede disfrazar la necesidad de cambios de ahora en adelante.

\section{BIBLIOGRAFÍA}

Amico, Fabian, "Argentina, 2002-2008: ciclo económico, inflación y crecimiento", in Oikos, año vi, núm. 10, Río de Janeiro, 2008, pp. 33-51.

, "Inflación de costos y conflicto distributivo en Argentina: un análisis de la etapa reciente", en $2^{\text {do }}$ Congreso Anual de la Asociación de Economía para el Desarrollo de la Argentina (AEDA), Buenos Aires, 2010.

Azpiazu, Daniel e Martín Schorr, Hecho en Argentina: industria y economia, 1976-2007, Buenos Aires, Siglo Veintiuno, 2010.

Barbosa-Filho, Nelson Henrique, "Inflation Targeting in Brazil: 1999-2006", in Epstein, Gerald y Erinc Yeldon (ed.), Beyond Inflation Targeting: Assessing the Impacts and Policy Alternatives, Cheltenham, Edward Elgar, 2009, pp. 139-157.

Bastos, Carlos Pinkusfeld Monteiro, "Inflação e Estabilização", in Fiori, José Luís e Carlos Medeiros (org.), Polarização Mundial e Crescimento, Petrópolis, Vozes, 2001, pp. 201-241. 
Bogdanski, Joel, Alexandre Antonio Tombini; Sérgio Ribeiro C. Werlang, "Implementing Inflation Targeting in Brazil", in Banco Central de Brasil Working Paper Series, num. 1, Brasília, Banco Central de Brasil, 2000.

Bresser Pereira, Luiz Carlos, Macroeconomia da Estagnação: Crítica da Ortodoxia Convencional no Brasil pós-1994, São Paulo, Editora 34, 2007. , Luiz Carlos, "Taxa de Câmbio, Doença Holandesa, e Industrialização”, in Cadernos FGV Projetos, 5(14), 2010, pp. 68-73.

Câmara, Alcino e Matias Vernengo, "The German Balance of Payment School and the Latin American Neo-Structuralists", in Rochon, Louis Philippe, Matias Vernengo (ed.), Credit, Interest Rates and the Open Economy, Cheltenham, Edward Elgar, 2001, pp. 143-159.

Canitrot, Adolfo, "A Experiência Populista de Redistribuição de Renda", in Bresser Pereira, Luiz Carlos (org.), Populismo Econômico, São Paulo, Nobel, 1991 (1975), pp. 11-36.

Cárdenas, Maurício, Eduardo Levy-Yeyati e Camila Henao, "Brookings Latin America Economic Perspectives", Brookings Latin America Initiative, 2010.

Céspedes, Luis Felipe, Ilan Goldfajn, Phil Lowe, Rodrigo Valdés, "Respuestas de política a shocks externos en Australia, Brasil y Chile", Economía Chilena, vol. 2, núm. 2, 2005, pp. 7-44.

Cunha, André Moreira y Andrés Ferrari, "A Argentina depois da Conversibilidade: um Caso de Novo-desenvolvimentismo?", en Revista de Economía Política, vol. 29, num. 1, São Paulo, 2009, pp. 2-23.

Curia, Eduardo, Teoría del modelo de desarrollo de la Argentina; las condiciones para su continuidad, Buenos Aires, Galerna, 2007.

Damill, Mario, Roberto Frenkel, "Las políticas macroeconómicas en la evolución reciente de la economía argentina", en Nuevos Documentos Cedes, num. 65, Buenos Aires, Cedes, 2009.

De Paula, Luiz Fernando, "O Tango Argentino", in Jornal Valor Econômico, 08 de abril, 2011.

Fabris, Julio Eduardo, Pablo Julio López y José Villadeamigo, "Efectos de la política cambiaria sobre precios y salarios: la experiencia argentina de la posconvertibilidad", en Congreso Anual de la Asociación de Economía para el Desarrollo de la Argentina (AEDA), Buenos Aires, 2009.

FMI, World Economic Outlook, Washington, Setembro, 2006.

Frenkel, Roberto y Martín Rapetti, "Five Years of Competitive and Stable Real Exchange Rate in Argentina, 2002-2007", en International Review of Applied Economics, vol. 22, num. 2, Londres, Routledge, 2008, pp. 215226. 
Gala, Paulo, "Propostas para uma Política Cambial com Foco no Desenvolvimento Econômico", in Ipea (org.), Macroeconomia para o Desenvolvimento: Crescimento, Estabilidade e Emprego-Livro 4, Projeto Perspectivas do Desenvolvimento Brasileiro, Rio de Janeiro, 2010, pp. 213-235.

Gonçalves, Reinaldo, "Continuidade, Antiliberalismo Instrumental e Ruptura. Liçóes da Argentina para o Brasil", in Revista da Sociedade Brasileira de Economia Politica, num. 14, Rio de Janeiro, 2004, pp. 135-147.

Levy Yeyati, Eduardo y Diego Valenzuela, La resurrección: historia de la poscrisis argentina, Buenos Aires, Sudamericana, 2007.

Mallon, Richard y Juan Vital Sorrouille, La politica económica en una sociedad conflictiva: el caso argentino, Buenos Aires, Amorrortu Editores, 1973.

Mecon, "Experiencias comparadas de estabilización en Argentina", en Analisis Econômico, núm. 4, Buenos Aires, 2005, pp. 34-38.

Mishkin, Frederic, Monetary Policy Strategy, Cambridge, Miт Press, 2007.

Modenesi, André y Salvador Werneck Vianna, "Avaliação da Política Monetária sob o Regime de Metas de Inflação", in Ipea (org.), Macroeconomia para o Desenvolvimento: crescimento, estabilidade e emprego, Livro 4, Projeto Perspectivas do Desenvolvimento Brasileiro, 2010, pp. 103-118.

Modenesi, André e Rui Lyrio Modenesi, "Quinze Anos de Rigidez Monetária no Brasil (1995-2008): uma Agenda de Pesquisa", in III Encontro da Associação Keynesiana Brasileira, São Paulo, 2010.

Nakano, Yoshiaki, "Aprimorar para Reduzir a Meta”, in Jornal Valor Econômico, 11 de janeiro, 2011.

Oreiro, José Luís e Carmen Feijó, "Desindustrialização: Conceituação, Causas, Efeitos e o Caso Brasileiro", in Revista de Economia Política, vol. 30, num. 2, São Paulo, 2010, pp. 219-232.

Oreiro, José Luis, "É o Novo Desenvolvimentismo?", in Jornal Valor Econômico, 27 de outubro, 2011.

Robinson, Joan, "A Review of The Economics of Inflation by BrescianiTurroni”, in Economic Journal, vol. 48, num. 191, Londres, Royal Economic Society, 1938, pp. 507-513.

Serrano, Franklin, "Juros, Câmbio e o Sistema de Metas de Inflação no Brasil", in Revista de Economia Política, vol. 30, num. 1, São Paulo, 2010, pp. 63-72.

Serrano, Franklin y Ricardo Summa, "Política Macroeconômica, Crescimento e Distribuição de Renda na Economia Brasileira dos Anos 2000", in Observatório da Economia Global, Textos Avulsos num. 6, Campinas, CECON-Unicamp, 2011. 
Serrano, Franklin y Sandra Ferreira, "Commodities, Câmbio e Inflação de Custos no Brasil, 1994-2009”, in Revista Versus Acadêmica, num. 4, Rio de Janeiro, CCJE-UfrJ, 2010, pp. 48-57.

Sturzenegger, Federico, Jeromin Zettelmeyer, Debt Defaults and Lessons from a Decade of Crises, Cambridge, MIT Press, 2006.

Versiani, Flávio R. y Wilson Suzigan, "O Processo Brasileiro de Industrialização: uma visão geral”, in X Congresso de História Econômica, Louvain, 1990. 